\title{
Derivada de funciones reales en el sentido de las distribuciones regulares
}

\author{
Derived from real functions in the sense of regular distributions
}

${ }^{1}$ Jhony Alfonso Chávez Delgado, ${ }^{2}$ Wilson Chanini Choquecota

\begin{abstract}
RESUMEN
El objetivo de este artículo es la generalización de la derivada de funciones ordinarias a derivada en el sentido de las distribuciones, de algunas funciones que no tienen derivada en el sentido ordinario, sin embargo, si estas funciones son tratados como distribuciones es posible extender el concepto de derivada de tal manera que cualquier número de derivadas pueda ser definida para estas funciones y verdaderas para cualquier distribución. En la generalización de la primera derivada de funciones ordinarias se estableció que mediante funciones localmente integrables son derivables en el sentido de las distribuciones. Asimismo, se emplearon los métodos lógico inductivo y deductivo respectivamente, el método deductivo se utilizó para justificar matemáticamente la existencia de funciones localmente integrables y el método inductivo para contrastar el funcionamiento de las funciones continuas o discontinuas utilizando ejemplos conocidos. En esta investigación los resultados que se obtuvo de la generalización de la derivada en el sentido de las distribuciones es que la derivada de la función valor absoluto es la función signo y la derivada de la función Heaviside es Delta de Dirac. Se concluye que la derivada en el sentido de las distribuciones es una generalización apropiada de la derivada clásica: cuando ambas existen; entonces coinciden como distribución y toda distribución es infinitamente derivable.
\end{abstract}

Palabras clave: primera derivada clásica, derivada débil, primera derivada generalizada.

\begin{abstract}
The purpose of this research work is the generalization of the derivative of ordinary functions to derivative in the sense of distributions, of some functions that have no derivative in the ordinary sense, however, if these functions are treated as distributions it is possible to extend The concept of derivative in such a way that any number of derivatives can be defined for these functions and green for any distribution. In the generalization of the first derivative of ordinary functions it is established that by means of locally integrable functions they are derivable in the sense of the distributions. Likewise, the inductive and deductive logic methods were used respectively; the deductive method was specifically to justify mathematically the existence of locally integrable functions and the inductive method to contrast the operation of continuous or discontinuous functions using known examples. One of the results obtained from the generalization of the derivative in the sense of the distributions is that derived from the absolute value function is the sign function and the derivative of the Heaviside function is Dirac Delta. It is concluded that the derivative in the sense of distributions is a specific generalization of the classical derivative: when both exist; then they coincide (as distribution) and all distribution is infinitely derivable.
\end{abstract}

Keywords: First classical derivative, Weak derivative, First generalized derivative or distribution.

\footnotetext{
${ }^{1}$ Universidad Nacional Jorge Basadre Grohmann. Tacna - Perú. E-mail: jchavezd@unjbg.edu.pe

${ }^{2}$ Universidad Nacional Jorge Basadre Grohmann. Tacna - Perú. E-mail: wilson_chanini@hotmail.com
} 


\section{INTRODUCCIÓN}

En el presente artículo de investigación, nuestro propósito es investigar la teoría de distribuciones para ahondar en el contenido de la generalización de la derivada de funciones reales a derivada en el sentido de las distribuciones regulares, enfocada en el método axiomático deductivo. En el análisis matemático, una distribución o función generalizada es un objeto matemático que generaliza la noción de función y la de medida. Además, la idea de distribución sirve para entender el concepto de derivada de todas las funciones localmente integrables y a entes aún más generales. Su aplicación es indispensable en muchos campos de la matemática, la física y la ingeniería. Por ejemplo, se utilizan en el análisis de Fourier para obtener soluciones generalizadas de ecuaciones en derivadas parciales. También juegan un papel muy importante en la electrodinámica cuántica y en el procesamiento de señales.

En el área de la matemática, se presentaron problemas en cuanto a generalizar el concepto de derivada en el sentido de las distribuciones, de funciones continuas y no continuas, cuyo propósito es formalizar y/o ordenar cada uno de los temas a desarrollarse con la rigurosidad lógica de las definiciones y las propiedades de la teoría de distribuciones, así como las contrastaciones mediante las ejemplificaciones para que sea más atractivo y de fácil acceso a los docentes universitarios, o más áreas, así como a los mismos estudiantes de ingeniería de las Universidades Nacionales.

Pero, en la limitación del problema nos preguntamos ¿bajo qué condiciones es posible establecer una generalización de la derivada clásica de funciones reales a derivada en el sentido de las distribuciones regulares? . En función de esa problemática, elegimos como propósito de establecer mediante la teoría de distribuciones el diferenciable y tienen su soporte contenido en un compacto de

Schawartz (1966). Cálculo de la derivada de funciones localmente integrables en el sentido de las distribuciones regulares. Desde el punto de vista metodológico se empleó en el desarrollo de la investigación el método axiomático deductivo que es la base de la construcción de cualquier disciplina matemática.

Este artículo contribuye a investigar las derivadas de las funciones reales en el sentido de las distribuciones regulares que ha sido estudiada por investigadores como Sobolev (1963); Schawart (1966); Adams (2006); (1992); Gouyon (1993) y otros.

\section{ESPACIO DE DISTRIBUCIONES ESCALARES}

\section{Funciones de prueba}

Los espacios serán definidos sobre un conjunto abierto $\Omega \subset i^{n}$

\section{Definición (Soporte compacto)}

Sea $\phi: i^{n} \rightarrow i$ una función real de variable vectorial. Se denomina soporte de $\phi$, denotado por $\operatorname{sop} \phi$, al conjunto $\operatorname{sop} \phi=\overline{\left\{x \in i^{n} / \phi(x) \neq 0\right\}}$

Gouyon (1972)

\section{Definición (función localmente integrable)}

Una función $f: \Omega \rightarrow \quad$ es localmente integrable si para todo conjunto compacto, $k \subset \Omega$ se tiene $\int_{k}|f(x)| d x<\infty$

Adams (1975).

\section{Definición (Espacio vectorial) $C_{0}^{\infty}(\Omega)$ )}

El espacio vectorial $C_{0}^{\infty}(\Omega)$ es el espacio de las funciones que son infinitamente

\section{Definición (Convergencia de funciones)}

Se dice que una sucesión de funciones $\left(\varphi_{n}\right)$ de $C_{0}^{\infty}(\Omega)$ converge para cero si, satisface las siguientes condiciones:

a) $\operatorname{sop}\left(\varphi_{n}\right) \subset k$, donde $k$ es un conjunto fijo en $\Omega \forall n=1,2,3, \ldots$

b) $\left(\varphi_{n}\right)$ converge uniformemente para cero en $\Omega$ , junto con todas las derivadas de cualquier orden. $D^{n} \varphi_{n} \rightarrow 0$, uniformemente $\forall \alpha \in ¥^{n}$ (multiindice)

Schwartz (1966).

\section{Definición (Funciones de prueba)}

El espacio vectorial $C_{0}^{\infty}(\Omega)$ con la noción de convergencia se denomina espacio de funciones de prueba.

Notación. El espacio de funciones de prueba se denota por $D(\Omega)$

Adams (1975).

\section{Definición (Derivada débil)}

Se dice que una función $u \in L_{l o c}^{1}$ posee derivada débil de orden 1 en $\Omega$, cuando existe una función $v \in L_{l o c}^{1}(\Omega)$ tal que 


$$
\begin{gathered}
\int_{\Omega} u(x) \psi^{\prime}(x) d x=-\int_{\Omega} v(x \psi(x) d x, \\
\forall \psi \in C_{0}^{\infty}(\Omega)
\end{gathered}
$$

Sobolev (1963)

\section{Definición (Funcional lineal)}

Sea $V$ un espacio vectorial sobre $i$. Se dice que una funcional lineal $\mathrm{T}$ sobre $\mathrm{V}$ es una transformación lineal de $\mathrm{V}$ en $i$.

\section{Definición (Distribución)}

Una distribución sobre $\Omega$ es una funcional $T: D(\Omega) \rightarrow i$ lineal y continua $D(\Omega)$. Tal espacio lo denotamos por $D^{\prime}(\Omega)$.

Es decir,

a) $\left\langle T, \psi_{1}+\psi_{2}\right\rangle=\left\langle T, \psi_{1}\right\rangle+\left\langle T, \psi_{2}\right\rangle$

donde $\psi_{1}, \psi_{2} \in D(\Omega)$

b) $\langle\alpha T, \psi\rangle=\alpha\langle T, \psi\rangle$

donde $\forall \psi \in D(\Omega), \forall \alpha \in$

c) si $\left(\psi_{v}\right)$ es una sucesion convergiendo para $\psi$, entonces $\left(\left\langle T \psi_{v}\right\rangle\right)$ converge $\operatorname{para}\left\langle T, \psi_{v}\right\rangle$

Schwartz (1966).

\section{Definición (Igualdad de distribuciones)}

Las Distribuciones $T_{1}$ y $T_{2}$ son iguales si y solo si $\left\langle T_{1}, \varphi\right\rangle=\left\langle T_{2}, \varphi\right\rangle \forall \varphi \in D(\Omega)$

\section{Lema (Dubois Raymond)}

Sea $f \in L_{l o c}^{1}(\Omega)$, si $T_{f}(\psi)=0$

$\forall \psi \in D(\Omega)$, entonces $f(x)=0$ en $\Omega$

(Sobolev, 1963)

Proposición (Funciones en $L_{l o c}^{1}(i)$ se identifican como distribución)

Si $f \in L_{l o c}^{1}(i)$, entonces el funcional $T_{f}$ definida por $D(\quad)$ mediante la expresión

$$
\left\langle T_{f}, \psi\right\rangle=\int f(x) \psi(x) d x, \forall \psi \in D(\quad)
$$

, es una distribución.

Espacio de $L^{p}([a, b])$
Sea $p \geq 1$. El espacio de funciones integrables en $[a, b]$, denotado por $L^{p}([a, b])$, se define $L^{p}([a, b])=\left\{\begin{array}{c}f:[a, b] \rightarrow \quad / f \text { medible y } \\ \int_{a}^{b}|f(x)|^{p} d x<\infty\end{array}\right\}$

\section{MATERIALES Y MÉTODOS}

En nuestro artículo es considerado material de estudio las funciones medibles con integral cuadrática en el sentido lebesgue y la teoría de distribuciones.

Se empleó en el desarrollo del artículo de investigación el método lógico deductivo, apoyado en la teoría axiomática, para la generalización de la derivada clásica de funciones reales a derivada en el sentido de las distribuciones. Se contrasto las demostraciones y definiciones usando ejemplos conocidos de la derivada de funciones continuas y no continúas en el sentido de las distribuciones.

\section{RESULTADOS}

Derivada débil de la función valor absoluto

Sea $u(x)=|x|$ y $\Omega=(-1,1), u \in L_{l o c}(\Omega)$

entonces la derivada débil es $\mathrm{v}(\mathrm{x})=\operatorname{sgn}(\mathrm{x})$.

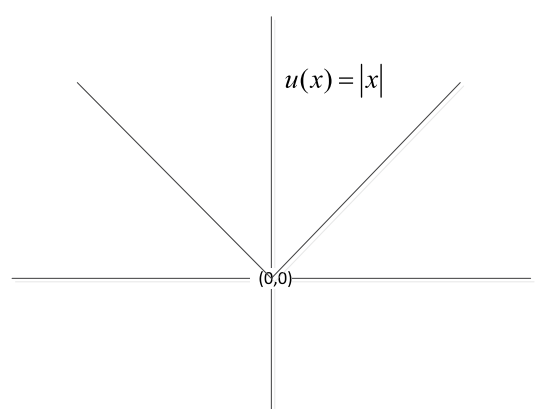

Fig.1. Función valor absoluto

Fuente: Elaboración propia

\section{Derivada de la distribución de la función cuadrática}

Sea $f(x)=a x^{2}+b x \forall x \in i, f \in L^{2}(\quad)$, entonces se cumple que

$$
\left\langle\mathscr{D} T_{f}, \psi\right\rangle=\langle 2 a x+b, \psi\rangle, \forall \psi \in \mathscr{D}\left({ }_{i}\right)
$$

\section{Derivada de la distribución de la función seno}

Sea $f(x)=\operatorname{sen}(a x), \forall x \in i, f \in L^{2}(\quad)$, entonces se cumple que

$\left\langle\mathscr{D} T_{f}, \psi\right\rangle=\langle a \cos (a x) \psi\rangle \forall \psi \in \mathscr{D}(\mathrm{i})$ 


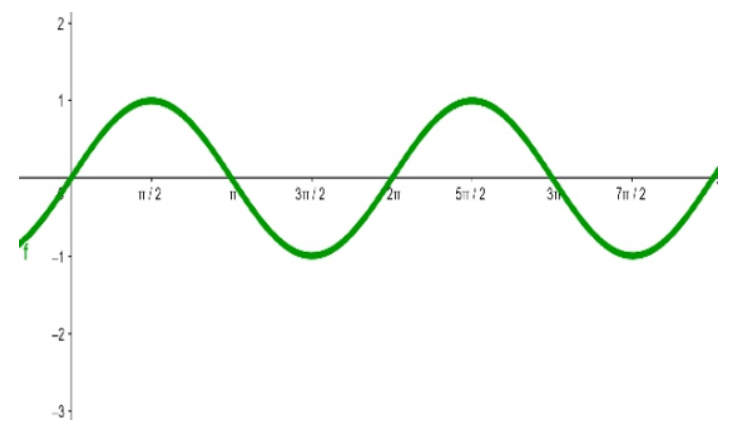

Fig.2. Función seno

Fuente: Elaboración propia

Derivada de la distribución de la exponencial

Sea $f(x)=e^{a x+b}, \forall x \in i, f \in L^{2}(\quad)$, entonces se cumple que

$\left\langle\mathscr{D} T_{f}, \psi\right\rangle=\left\langle a e^{a x+b}, \psi\right\rangle, \forall \psi \in \mathscr{D}(\mathrm{i})$

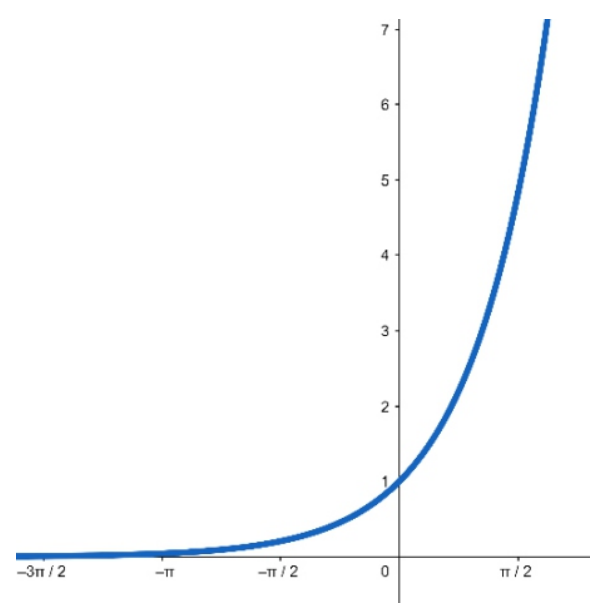

Fig.3. Función exponencial

Fuente: Elaboración propia

Derivada de la distribución de la función Heaviside

Sea $H(x)=\left\{\begin{array}{l}0, \text { si } x<0 \\ 1, \text { si } x>0\end{array}, \forall x \in \mathrm{i}\right.$,

$\Omega=\langle-1,1\rangle, f \in L_{l o c}(\Omega)$ (Función Heaviside o escalón unitario)

Entonces se cumple que:

$\left\langle\mathscr{D} T_{H}, \psi\right\rangle=\langle\delta, \psi\rangle \forall \psi \in \mathscr{D}(\Omega)$

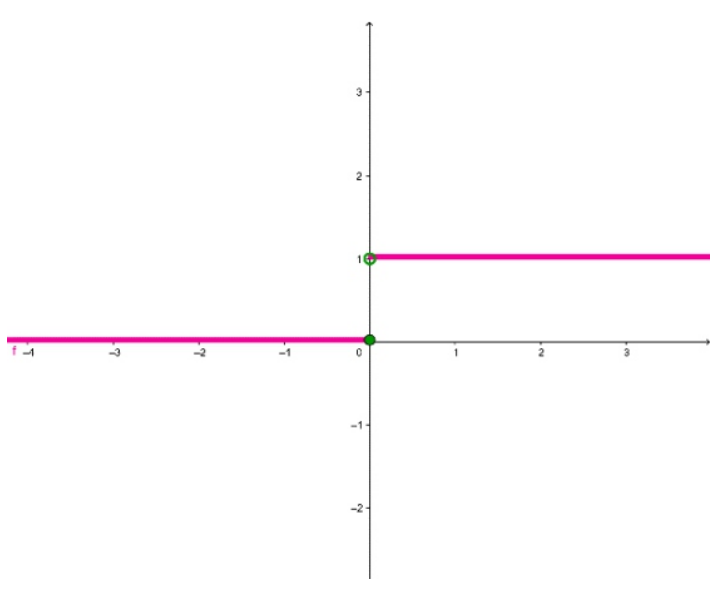

Fig.4. Función Heaviside Fuente: Elaboración propia

\section{DISCUSIÓN}

\section{Proposición (Derivada débil de la función valor} absoluto)

\section{Prueba}

Sea $u(x)=|x|_{\text {y }} \Omega=(-1,1), u \in L_{l o c}(\Omega)$, entonces la derivada débil es $\mathrm{v}(\mathrm{x})=\operatorname{sgn}(\mathrm{x})$.

\section{Prueba}

Se debe calcular

$\int_{-1}^{1}|x| \varphi^{\prime}(x) d x \quad \forall \varphi \in C_{0}^{+\infty}(\Omega)$

En efecto, Integrando por partes, se obtiene

$$
\begin{aligned}
& \int_{-1}^{1}|x| \varphi^{\prime}(x) d x=-\int_{-1}^{0} x \varphi^{\prime}(x) d x+\int_{0}^{+1} x \varphi^{\prime}(x) d x \\
& \left.=-x \varphi(x)]_{-1}^{0}+\int_{-1}^{0} \varphi(x) d x+x \varphi(x)\right]_{0}^{1}-\int_{0}^{1} \varphi(x) d x \\
& =-\int_{-1}^{0} \operatorname{sgn}(x) \varphi(x) d x-\int_{0}^{1} \operatorname{sgn}(x \phi(x) d x \\
& =-\int_{-1}^{1} \operatorname{sgn}(x) \varphi(x) d x
\end{aligned}
$$

Luego se concluye que $\mathrm{v}(\mathrm{x})=\operatorname{sgn}(\mathrm{x})$ en $v \in L_{l o c}^{1}(\Omega) \mathrm{y}$

$\int_{-1}^{1}|x| \varphi^{\prime}(x) d x==-\int_{-1}^{1} \operatorname{sgn}(x) \varphi(x) d x \quad \forall \varphi \in C_{0}^{\infty}(\Omega)$ 
Por lo tanto, la derivada débil de $u(x)=|x|$ en $\Omega=(-1,1)$ es $v(x)=\operatorname{sgn}(x)$.

\section{Proposición (Derivada de la distribución de la} función cuadrática)

Sea $f(x)=a x^{2}+b x \forall x \in i, f \in L^{2}(\quad)$, entonces se cumple que

$\left\langle\mathfrak{D} T_{f}, \psi\right\rangle=\langle 2 a x+b, \psi\rangle, \forall \psi \in \mathscr{D}\left({ }_{i}\right)$

Por lo tanto $D T_{f}=2 a x+b$

Sea el conjunto abierto $\Omega=i$. Luego por definición de la derivada de una distribución se tiene:

$$
\begin{aligned}
\left\langle\mathfrak{D} T_{f}, \psi\right\rangle & =\left(\mathfrak{D} T_{f}\right)(\psi) \\
& =(-1)^{1}\left\langle T_{f}, \psi\right\rangle \\
& =-\left\langle T_{f}, \psi^{\prime}\right\rangle \\
& =-\int_{-\infty}^{+\infty} f(x) \psi^{\prime}(x) d x
\end{aligned}
$$

Aplicando integración por partes en (1), se obtiene

$$
\begin{aligned}
& =-\int_{-\infty}^{+\infty}\left(a x^{2}+b x\right) \psi^{\prime}(x) d x \\
& =-\left[\left(a x^{2}+b x\right) \psi(x)\right]_{-\infty}^{+\infty}+\int_{-\infty}^{+\infty}(2 a x+b \psi(x) d x \\
& \left.=-\left(a x^{2}+b x\right)\right]_{-\infty}^{+\infty}+\int_{-\infty}^{+\infty}(2 a x+b) \psi(x) d x
\end{aligned}
$$

Entonces, por definición de soporte compacto en (2) resulta que

$$
\left\langle\mathfrak{D} T_{f}, \psi\right\rangle=\int_{-\infty}^{+\infty}(2 a x+b) \psi(x) d x
$$

Por lo tanto

$$
\left\langle\mathfrak{D} T_{f}, \psi\right\rangle=\langle 2 a x+b, \psi\rangle, \forall \psi \in \mathscr{D}\left({ }_{i}\right)
$$

\section{Proposición (Derivada de la distribución de la función seno)}

Sea $f(x)=\operatorname{sen}(a x), \forall x \in i, f \in L^{2}(\quad)$, entonces se cumple que

$$
\left\langle\mathfrak{D} T_{f}, \psi\right\rangle=\langle a \cos (a x) \psi\rangle \forall \psi \in \mathscr{D}(\mathrm{i})
$$

\section{Prueba}

Sea el conjunto abierto $\Omega=i$. Luego por definición de la derivada de una distribución se tiene

Aplicando el Teorema de integración por partes en (5), se obtiene

$$
\begin{aligned}
\left\langle\mathfrak{D} T_{f}, \psi\right\rangle & =(-1)^{1}\left\langle T_{f} \psi^{\prime}\right\rangle \\
& =-\int_{-\infty}^{+\infty} f(x) \psi^{\prime} d x
\end{aligned}
$$

Aplicando el teorema de la integración por partes en (3), se obtiene

$$
\begin{aligned}
& =-\int_{-\infty}^{+\infty} \operatorname{sen}(a x) \psi^{\prime} d x \\
& =-\left[\left.\operatorname{sen}(a x) \psi(x)\right|_{-\infty} ^{+\infty}-\int_{-\infty}^{+\infty} a \operatorname{sen}(a x \psi(x) d x \mid]\right. \\
& =-\left.\operatorname{sen}(a x) \psi(x)\right|_{-\infty} ^{+\infty}+\int_{-\infty}^{+\infty} a \cos (a x) \psi(x) d x(4)
\end{aligned}
$$

Entonces, por la definición de soporte compacto en (4) resulta que

$$
\begin{aligned}
\left\langle\mathfrak{D} T_{f}, \psi\right\rangle & =\int_{-\infty}^{+\infty} a \cos (a x) \psi(x) d x \\
& =\langle a \cos (a x), \psi\rangle
\end{aligned}
$$

Por lo tanto

$$
\left.\mathscr{D} T_{f}=a \cos (a x) \forall \psi \in \mathscr{D}_{i}\right)
$$

\section{Derivada de la distribución de la función exponencial}

Sea $f(x)=e^{a x+b}, \forall x \in i, f \in L^{2}(\quad)$, entonces se cumple que

$$
\left\langle\mathscr{D} T_{f}, \psi\right\rangle=\left\langle a e^{a x+b}, \psi\right\rangle, \forall \psi \in \mathscr{D}(\mathrm{i})
$$

\section{Prueba}

Sea el conjunto abierto $\Omega \subset i$. Luego por definición de la derivada de una distribución se tiene:

$$
\begin{aligned}
\left\langle\mathfrak{D} T_{f}, \psi\right\rangle & =(-1)^{1}\left\langle T_{f}, \psi^{\prime}\right\rangle \\
& =-\int_{-\infty}^{+\infty} f(x) \psi^{\prime}(x) d x
\end{aligned}
$$




$$
\begin{aligned}
& =-\int_{-\infty}^{+\infty} e^{a x} \psi^{\prime}(x) d x \\
& =-\left[\left.e^{a x+b} \psi(x)\right|_{-\infty} ^{+\infty}-\int_{-\infty}^{+\infty} a e^{a x+b} \psi(x) d x \mid\right] \\
& =-\left.e^{x} \psi(x)\right|_{-\infty} ^{+\infty}+\int_{-\infty}^{+\infty} a e^{a x+h} \psi(x) d x \\
& \left.=-\left[e^{\infty} \psi(\infty)-e^{-\infty} \psi(\infty)+\int_{-\infty}^{+\infty} a e^{a x+b} \psi(x) d x\right]\right]
\end{aligned}
$$

Entonces, por la definición de soporte compacto en (6) resulta que

$$
\begin{aligned}
\left\langle\mathcal{D} T_{f}, \psi\right\rangle & =\int_{-\infty}^{+\infty} a e^{a x} \psi(x) d x \\
& =\left\langle a e^{a x}, \psi\right\rangle
\end{aligned}
$$

Por lo tanto

$$
\mathfrak{D} T_{f}=a e^{a x+b} \forall \psi \in \mathcal{D}(\Omega)
$$

\section{Proposición (Derivada de la distribución de la función Heaviside)}

Sea $H(x)=\left\{\begin{array}{l}0, \text { si } x<0 \\ 1, \text { si } x>0\end{array}, \forall x \in \mathrm{i}\right.$,

$\Omega=\langle-1,1\rangle, f \in L_{l o c}(\Omega)$

Entonces se cumple que:

$\left\langle\mathscr{D} T_{H}, \psi\right\rangle=\langle\delta, \psi\rangle \forall \psi \in \mathscr{D}(\Omega)$

\section{Prueba}

Sea el conjunto abierto $\Omega \subset i$. Luego por definición de la derivada de una distribución se tiene:

$$
\begin{aligned}
& \langle\mathfrak{D} H, \psi\rangle=\left\langle\frac{d H}{d x}, \psi\right\rangle=\left\langle\mathfrak{D}_{H}, \psi\right\rangle=(-1)^{1}\left\langle T_{H}, \psi^{\prime}\right\rangle \\
& -\left\langle T_{f}, \psi^{\prime}\right\rangle=-\int_{-\infty}^{+\infty} H(x) \psi^{\prime}(x) d x \\
& =-\int_{i} H(x) \psi^{\prime}(x) d x \\
& \left.=-\left[\int_{-\infty}^{0} H(x) \psi^{\prime}(x) d x+\int_{0}^{\infty} H(x) \psi^{\prime}(x) d x\right]\right] \\
& =-\left(\int_{-\infty}^{+\infty} 0 . \psi^{\prime}(x) d x+\int_{0}^{\infty} 1 \psi^{\prime}(x) d x\right) \\
& =-\int_{0}^{+\infty} \psi^{\prime}(x) d x \\
& =-\int_{0}^{+\infty} \frac{d \psi(x)}{d x} \\
& =-\int_{0}^{+\infty} d \psi \\
& =-(\psi(\infty)-\psi(0))
\end{aligned}
$$

Entonces, por la definición de soporte compacto en (7) resulta que

$$
=-(0-\psi(0))=\psi(0)
$$

Por lo tanto

$$
\begin{aligned}
& \langle\mathscr{D H}, \psi\rangle=\psi(0)=\delta(\psi)=\langle\delta, \psi\rangle \\
& H^{\prime}=\delta \forall \psi \in \mathscr{D}(\Omega)
\end{aligned}
$$

\section{CONCLUSIONES}

Las funciones medibles e integrables con integral cuadrática en el sentido Lebesgue poseen derivadas en el sentido de las distribuciones.

Las funciones localmente integrables poseen derivadas en el sentido de las distribuciones.

\section{REFERENCIAS BIBLIOGRÁFICAS}

ADAMS, R. Sobolev spaces. Academic Press, Inc. London. 1975

APÓSTOL, M. Análisis matemático.2. a ed, 2006. BRINTON, G. Calculo en varias variables, $11^{\mathrm{a}} \mathrm{ed}$. México, 2005.

CORNEJO, M. \& Villalobos, E. \& Quintanilla A. Métodos de solución de ecuaciones diferenciales y aplicaciones. 1. ${ }^{a}$ ed. México ,2008.

GOUYON, R. Integración y distribuciones. 1972

JAMES, G. Matemáticas avanzadas para ingeniería, 2. ed. México, 2002

LERAY, J. \& Ohya, Y. Équations et systèmes nonlinéaires, hyperboliques nonstricts. Math. Annalen 170, 167-205.1967

NAGLE, R. Ecuaciones diferenciales y problemas con valores en la frontera. 4. ${ }^{\text {a }}$ ed. 2005

SOBOLEV, S. Some applications of functional analysis in mathematical physics. Amer. Math. Soc. 1963

SCHWARTZ, L. Mathematics for the Physical Sciences”. Edición: Dover.1966 